\title{
Generalisations of the Ginsparg-Wilson relation and a remnant of supersymmetry on the lattice
}

\author{
Georg Bergner* \\ Theoretisch-Physikalisches Institut, Universität Jena, D-07743 Jena, Germany \\ E-mail: g.bergneretpi.uni-jena.de
}

\section{Falk Bruckmann}

Institut für Theoretische Physik, Universität Regensburg, D-93040 Regensburg, Germany

E-mail: falk.bruckmann@physik.uni-regensburg.de

\section{Jan M. Pawlowski}

Institut für Theoretische Physik, Universität Heidelberg, D-69120 Heidelberg, Germany

E-mail: j.pawlowski@thphys.uni-heidelberg.de

\begin{abstract}
We introduce a lattice symmetry relation for field theories with general linear symmetries. For chiral symmetry the well-known Ginsparg-Wilson relation is reproduced. The new relation encodes the remnant of the original symmetry on the lattice and guides the construction of invariant lattice actions. We apply this approach to lattice supersymmetry. There, an additional constraint has to be satisfied which originates in the derivative operator in the symmetry transformations. As a consequence the non-local SLAC derivative operator appears in the lattice transformation. Despite this non-local form we show how local solutions for quadratic actions can be found. For interacting theories the relation in general leads to a non-polynomial action that can be reduced to a finite polynomial order only under certain conditions.
\end{abstract}

The XXVI International Symposium on Lattice Field Theory

July 14-19 2008

Williamsburg, Virginia, USA

* Speaker. 


\section{Introduction}

One of the basic problems of lattice simulations of quantum field theory is the continuum limit. The specific form of the lattice action governs not only the renormalisation; in practice it also decides, whether it is possible to approach the continuum theory sufficiently fast for the numerical resources at hand.

To preserve or recover the symmetries of the continuum theory is an essential part of the continuum limit. In some important cases continuum symmetries are necessarily broken on the lattice. This applies in particular to space-time symmetries, as the lattice introduces a hard breaking of the rotational and translational invariance of the continuum. Supersymmetry is another example for this situation that indeed relates to the aforementioned space-time symmetry. On the technical level this manifests itself in the failure of the Leibniz rule on the lattice [1], which makes it impossible to find a local lattice action preserving the full supersymmetry of an interacting theory. With no realisation of supersymmetry at finite lattices, the continuum limit is thus very hard to control.

Related problems are the representation of the continuum anomalies on the lattice and the different possible realisations of symmetry operators on the lattice, which can not all be expected to lead to the correct continuum limit. The naive realisation of chiral symmetry on the lattice, for example, can only be achieved at the cost of an unwanted doubling of the degrees of freedom, as stated by the Nielsen-Ninomiya theorem [2]. For symmetries broken on the lattice, the symmetric continuum theory may be achieved by fine-tuning the parameters of the action. This type of renormalisation procedure, however, is numerically rather expensive and requires knowledge of physical quantities that should actually be a result of the numerical calculations.

The approach of Ginsparg and Wilson [3] to chiral symmetry resolves this fine-tuning problem within a renormalisation group setting. The procedure is based on a block spin transformation, i.e. a mapping from the continuum to the lattice (or from a finer lattice to a coarser one). This guarantees the minimal amount of symmetry breaking at each blocking step and also ensures a definite interpretation of the lattice observables in terms of continuum quantities and the correct continuum limit. In principle this setting can also be used to calculate an effective lattice action, but explicit solutions are calculable only in very special cases. It is, however, possible to obtain the implications of the continuum symmetries purely on the level of the effective lattice action: continuum symmetries are translated into lattice relations that can be interpreted as deformed lattice symmetries. Lattice implementations which obey these relations ensure the correct symmetries in the continuum limit. Moreover, the deformed symmetry operators provides us with the correct realisation of the continuum symmetry on the lattice. Such an approach is closely related to the renormalisation group studies in the continuum. The lattice is in that case just a special regulator of the theory and the lattice symmetry relations correspond to the quantum master equations or modified Slavnov-Taylor identities, for a review see [4].

Here we consider the blocking procedure for general continuum theories with general linear symmetries [5]. This systematic approach enables us to derive a relation for lattice actions with remnant lattice (super)symmetry. Locality problems of the naive implementation of (super)symmetry might be cured by an appropriately chosen blocking term leading to a deformed lattice (super)symmetry. We discuss both quadratic theories, revisiting the chiral case as a pedagogical example, as well as interacting theories. Evidently, the main obstructions for the solutions of the symmetry relation will be space-time locality and a polynomial nature in the fields. 


\section{Blocking transformation}

The blocking transformation specifies the map from the continuum theory onto the lattice. In particular this map entails how lattice symmetry transformations derive from their continuum analogues, and which symmetry relation the blocked lattice action has to satisfy.

The first step of the blocking procedure is an averaging of the continuum fields $\varphi(x)$ around each lattice point $n$, done with an averaging function $f(x)$,

$$
\Phi_{n}[\varphi]:=\int d x f\left(x-x_{n}\right) \varphi(x),
$$

that is typically peaked around zero. In this way we have defined averaged fields $\Phi_{n}$ with an integer lattice index $n$. This averaging can easily be extended to more than one dimension.

The second step introduces a blocking matrix $\alpha$ that connects the averaged fields $\Phi_{n}$ to the lattice fields $\phi_{n}$ via a Gaussian factor,

$$
e^{-S[\phi]}:=\frac{1}{\operatorname{SDet}^{1 / 2} \alpha} \int d \varphi e^{-\frac{1}{2}(\phi-\Phi[\varphi])_{n} \alpha_{n m}(\phi-\Phi[\varphi])_{m}} e^{-S_{\mathrm{cl}}[\varphi]},
$$

which can be read as smearing in field space. It defines a blocked lattice action $S[\phi]$ for a given continuum action $S_{\mathrm{cl}}[\varphi]$ and blocking kernel $\alpha$.

This prescription has a simple interpretation if $f\left(x-x_{n}\right)$ approaches $\delta\left(x-x_{n}\right)$ and the eigenvalues of $\alpha$ diverge in the continuum limit: then the lattice action becomes the continuum action. The first demand is actually a necessary one in order to approach the continuum, since $f$ governs the resolution of the lattice. For more general $\alpha$ 's one has to investigate the generating functional with the action $S[\phi]$ defined above, because this quantity determines the meaning of the observables in the $\phi$ theory. Performing Gaussian integrations one gets an exact agreement,

$$
\int d \phi e^{-S[\phi]+J \phi}=e^{-J \alpha^{-1} J} \int d \varphi e^{-S_{\mathrm{cl}}[\varphi]+J \Phi[\varphi]},
$$

up to the $J$-dependent prefactor that can be easily calculated knowing $\alpha$.

The two steps explained above will be investigated w.r.t. their effect on lattice symmetries in the next two sections. We shall see that for global symmetries such as chiral symmetry the first step is trivial. For a general space-time-dependent symmetry, and especially supersymmetry, also the first step is non-trivial and leads to obstructions.

\section{Lattice symmetry: the blocked generator ...}

Here we consider a continuum action $S_{\mathrm{cl}}[\varphi]$ that is invariant under an infinitesimal continuum variation $\tilde{\delta} \varphi^{i}=\varepsilon \tilde{M}^{i j} \varphi^{j} . \varepsilon$ is an infinitesimal parameter, bosonic or fermionic, and the superscript $i$ labels internal indices and species of fields. For example, in the case of chiral symmetry $\varphi^{i}$ are the components of a spinor, and $\tilde{M}$ is a (global) chiral rotation.

After the averaging step (2.1) this symmetry must act only among the discrete fields labelled by $n$. This means that the transformation must be 'pulled outside' of the averaging procedure as

$$
\int d x f\left(x-x_{n}\right) \varepsilon \tilde{M}^{i j} \varphi^{j}(x)=\varepsilon M_{n m}^{i j} \Phi_{m}\left[\varphi^{j}\right]=\varepsilon M_{n m}^{i j} \int d x f\left(x-x_{m}\right) \varphi^{j}(x) \quad \forall \varphi^{j}(x),
$$

to arrive at a lattice transformation $M$ and the corresponding variation $\delta \phi_{n}^{i}=\varepsilon M_{n m}^{i j} \phi_{m}^{j}$. 
It is trivial to compute $M$ for a symmetry that acts only on multiplet indices and (3.1) was therefore not taken into account in the discussions of chiral symmetry. Starting with a continuum transformation this procedure defines a corresponding lattice counterpart. Note, however, that for space-time dependent symmetries one cannot find such an $M$ for every $\tilde{M}$ and $f$. Then (3.1) constitutes an additional constraint for $\tilde{M}, M$ and $f$ and is not a mere definition of $M$. This applies in particular to the derivative operator in the continuum supersymmetry $\tilde{M}$, a problem ${ }^{1}$ to which we will come back in section 6 .

We conclude that an invariance under the naive symmetry transformations $\delta \phi$ is the first guess for the resulting lattice symmetry. However, in order to calculate the full effect of the blocking on the symmetry one must also include the blocking matrix $\alpha$ as done in the next section.

\section{4. .... and the symmetry relation}

The symmetry relation for the lattice action is derived from applying an infinitesimal transformation on the lattice fields, $\phi$ in (2.2). This transformation can be absorbed within a symmetry transformation of the continuum fields $\varphi$ on the rhs. of (2.2) using the additional constraint. More details of the calculation can be found in [5]. We finally arrive at the following relation

$$
M_{n m}^{i j} \phi_{m}^{j} \frac{\delta S}{\delta \phi_{n}^{i}}=\left(M \alpha^{-1}\right)_{n m}^{i j}\left(\frac{\delta S}{\delta \phi_{m}^{j}} \frac{\delta S}{\delta \phi_{n}^{i}}-\frac{\delta^{2} S}{\delta \phi_{m}^{j} \delta \phi_{n}^{i}}\right)+(\mathrm{S} \operatorname{Tr} M-\mathrm{S} \operatorname{Tr} \tilde{M}) .
$$

The trace part $\operatorname{STr} \tilde{M}$ takes care of an infinitesimal change of the measure $d \varphi$, hence the last term represents the difference between continuum and lattice anomalies.

The relation (4.1) entails the deformation of the continuum symmetry on the lattice due to the chosen blocking kernel $\alpha^{-1}$. For symmetric blocking kernels the rhs. of (4.1) vanishes and the lattice theory respects the symmetry generated by $M, \delta S=\varepsilon M \phi \delta S / \delta \phi=0$. More specifically, a symmetric part $\alpha_{S}^{-1}$ does not contribute to 4.1 if it satisfies

$$
M \alpha_{S}^{-1} \pm\left(M \alpha_{S}^{-1}\right)^{T}=0
$$

(the minus sign applies when the symmetry transforms fermions into fermions). It is therefore the symmetry breaking part of the blocking kernel that is responsible for the difference between the naive and the deformed lattice symmetry, by generating non-linear terms on the rhs. of (4.1). We emphasise that the averaging function $f$, which is a key ingredient of the mapping onto the lattice, appears in this relation only in terms of the naive lattice symmetry operator $M$.

\section{Quadratic lattice actions and chiral symmetry}

To achieve a better understanding of the above relation (4.1), we discuss it in the simplest case of quadratic actions. Although this seems to be a rather trivial case, it already includes the well known Ginsparg-Wilson (GW) relation for chiral symmetry. There the Dirac action, relevant for this symmetry, is quadratic for a given gauge background.

Evaluating our relation (4.1) for a quadratic lattice action, $S=\frac{1}{2} \phi_{n}^{i} K_{n m}^{i j} \phi_{m}^{j}$, we obtain a matrix equation from the terms quadratic in the fields (for the field-independent trace part see [f]]),

$$
M^{T} K \pm\left(M^{T} K\right)^{T}=K^{T}\left(M \alpha^{-1}\right)^{T} K \pm\left(K^{T}\left(M \alpha^{-1}\right)^{T} K\right)^{T} .
$$

Again, the minus sign applies when the symmetry transforms fermions into fermions.

\footnotetext{
${ }^{1}$ This constraint has been mentioned without further investigation in 6$]$.
} 
Now we discuss the specific case of chiral symmetry with symmetry operator $\tilde{M}, M \sim \gamma_{5}$ and the simplest blocking kernel $\alpha_{n m}=\frac{1}{a} \delta_{n m}$, that is diagonal in lattice sites and indeed diverging in the continuum limit. Then the matrix relation (5.1) is nothing but the Ginsparg-Wilson relation $\left\{\gamma_{5}, D\right\}=a D \gamma_{5} D[3]$. Furthermore (5.1) can be rewritten as

$$
M_{\mathrm{def}}^{T} K \pm\left(M_{\mathrm{def}}^{T} K\right)^{T}=0, \quad \text { with } \quad M_{\mathrm{def}}=M\left(1-\alpha^{-1} K\right) .
$$

Eq. (5.2) entails the invariance of the action under a transformation generated by $M_{\text {def }}$. In other words, the invariance of the continuum action always implies an invariance of the lattice action. There are, however, other conditions for $M_{\text {def }}$ to qualify as a lattice symmetry: Clearly $M_{\text {def }}$ has to approach its continuum counterpart in the continuum limit. Furthermore, $M_{\text {def }}$ must be local to define a proper lattice symmetry, in order to have lattice artefacts of the symmetry under control in the continuum limit. For non-local lattice artefacts the continuum limit is at stake. We conclude that the freedom to choose a blocking kernel must be utilised to find a local deformed symmetry. We will denote only invariances satisfying the conditions explained above as lattice symmetry. In turn, non-local invariances should not be seen as lattice symmetries.

In the case of chiral symmetry with blocking kernel $\alpha_{n m}=\frac{1}{a} \delta_{n m}$ one arrives at deformed $\gamma_{5}$ 's, now depending on the Dirac operator and hence the background field, cf. [7]. The locality conditions excludes for instance Wilson fermions as a solution for the chiral symmetry. Indeed, locality is the main issue about solutions of the Ginsparg-Wilson relation. This problem already occurs for quadratic theories and we will face it again for supersymmetry below.

\section{A derivative operator for lattice supersymmetry}

Now we return to the additional constraint (3.1) with $\tilde{M}$ containing the derivative operator, as is the case for supersymmetry. The constraint entails, that the averaged derivative of any continuum field must be represented by a linear combination of averaged fields living on the lattice sites only. The corresponding coefficients constitute the lattice derivative operator $\nabla$. On the averaging function $f$ itself and transformed into Fourier-space the constraint reads

$$
\left[\nabla\left(p_{k}\right)-i p_{k}\right] f\left(p_{k}\right)=0 \quad \forall p_{k}=\frac{2 \pi}{L} k, \quad k \in \mathbb{Z}, \quad \text { with } \quad \nabla\left(p+\frac{2 \pi}{a}\right)=\nabla(p) .
$$

Eq. (6.1) can only be satisfied if for all momenta $p_{k}$ either the Fourier component of the averaging function, $f\left(p_{k}\right)$, or the difference between the continuum and the lattice derivative operator vanishes. If $f\left(p_{k}\right)$ vanishes only outside the first Brillouin zone, the only solution for $\nabla$ is the non-local SLAC-derivative [8], which can nevertheless be useful in some low dimensional models [9].

If $f\left(p_{k}\right)$ also vanishes for momenta inside the first Brillouin zone, other lattice derivative operators are allowed, but the averaging function $f$ then introduces an effective cutoff below $2 \pi / a$ [5]. From a blocking perspective $f$ then generates less independent blocked fields than present on the lattice. Note also that the non-local derivative operator does not necessarily appear in the action or the lattice symmetry operator, it is just included in the naive lattice transformations $M$.

\section{Free supersymmetry}

Now we have all ingredients to discuss supersymmetry on the lattice. For simplicity we consider supersymmetric quantum mechanics (SUSYQM). For supersymmetric quantum mechanics the continuum multiplet $\varphi$ consists of bosonic fields $\chi$ and $F$ (the latter being non-dynamical) plus fermionic fields $\psi$ and $\bar{\psi}$, which are transformed into each other under supersymmetry. 
The field content on the lattice and the naive lattice transformations

$$
\delta\left(\begin{array}{l}
\chi \\
F \\
\psi \\
\bar{\psi}
\end{array}\right)_{n}=\left(\begin{array}{cccc}
0 & 0 & -\bar{\varepsilon} & \varepsilon \\
0 & 0 & -\bar{\varepsilon} \nabla & -\varepsilon \nabla \\
-\varepsilon \nabla & -\varepsilon & 0 & 0 \\
\bar{\varepsilon} \nabla & -\bar{\varepsilon} & 0 & 0
\end{array}\right)_{n m}\left(\begin{array}{c}
\varphi \\
F \\
\psi \\
\bar{\psi}
\end{array}\right)_{m}=(\varepsilon M+\bar{\varepsilon} \bar{M})_{n m}^{i j} \phi_{m}^{j},
$$

are analogous to the continuum ones. The infinitesimal parameters $\varepsilon$ and $\bar{\varepsilon}$ are Grassmannian and the SLAC-derivative, $\nabla$, replaces the continuum derivative as discussed above. As a general ansatz, the quadratic (off-shell) lattice action has the kernel

$$
K_{n m}^{i j}=a\left(\begin{array}{cccc}
-\square_{n m} & -m_{b, n m} & 0 & 0 \\
-m_{b, n m} & -I_{n m} & 0 & 0 \\
0 & 0 & 0 & \left(\hat{\nabla}-m_{f}\right)_{n m} \\
0 & 0 & \left(\hat{\nabla}+m_{f}\right)_{n m} & 0
\end{array}\right) .
$$

Translation invariance demands that all of these matrices are circulant, i.e. they commute. The matrices $I, \square, m_{b, f}$ are symmetric, whereas $\hat{\nabla}$ is antisymmetric and in the continuum limit they need to approach $1, \partial^{2}, m$ and $\partial$, respectively.

Together with the blocking kernel, these matrices are subject to our relation (5.1). First we notice that a vanishing $\alpha^{-1}$, or a symmetric one according to eq. (4.2), yields a deformed symmetry equal to the naive one. These are non-local due to the presence of the SLAC operator.

Hence we must use a nontrivial blocking kernel for generating local lattice actions with local symmetries. First we choose $\alpha^{-1}$ diagonal in lattice indices, as for the Ginsparg-Wilson relation, and - due to symmetry reasons - symmetric in the bosonic sector and antisymmetric in the fermionic sector. A solution of the coefficient matrices in the action (7.2) in terms of the blocking kernel and the SLAC-derivative $\nabla$ is possible and non-trivial, see [5]. In particular, the lattice derivative $\hat{\nabla}$ in the action is proportional to $\nabla$ with a prefactor similar to a massive propagator (const. $\left.^{2}-\nabla^{2}\right)^{-1}$. In the continuum similar expressions lead to an exponential decay for large distances. On the lattice, however, the corresponding behaviour is spoilt by terms decaying only algebraically, exactly because $\nabla$ is finite at the boundary of the first Brillouin zone [f]].

Since the blocking kernel $\alpha$ has no direct physical implication, we now adjust $\alpha^{-1}$ according to a local action and local deformed supersymmetry. With a general ansatz for $M_{\text {def }}$ and after some computations we are left with a relation $\hat{\nabla}=I \nabla[$ [ $]$. As $\hat{\nabla}$ and $I$ appear in the lattice action and in the deformed symmetry, they must be local. This implies that $I$ vanishes together with all its derivative at the boundary of the Brillouin zone. In this delicate way a decay of the Dirac operator $\hat{\nabla}$ stronger than algebraic (but not exponential) can be achieved.

\section{Interacting systems}

One of the important properties of our symmetry relation (4.1) is its validity for interacting theories. Therefore, we can embark on constructing a local interacting supersymmetric theory on the lattice in our approach.

Beyond second order, however, the non-linearity of this relation starts to play an important role. Since it connects different orders of fields, solutions of the symmetry relation are generically non-polynomial. This is not unexpected since the blocked action is comparable to an effective 
action. In order to truncate the tower of interactions, a necessary condition is (in matrix notation)

$$
\frac{\partial S^{\max }}{\partial \phi} M \alpha^{-1} \frac{\partial S^{\max }}{\partial \phi}=0
$$

where $S^{\max }$ denotes the part of the action with the maximal order in the fields. This means that this highest $S^{\max }$ does not depend on the entire field multiplet. For SUSY, a purely bosonic $S^{\max }$ suffices as $M$ mixes bosons with fermions. We have illustrated this peculiar situation by virtue of an exact solution of interacting SUSYQM restricted to constant fields, which depending on the choice of $\alpha$ is either logarithmic or polynomial [5], for chiral theories see [10]. We stress that eq. (8.1) represents only one relation of those that follow from our relation (4.1) when ordered according to the powers of fields.

\section{Summary}

In this brief survey we have shown that symmetries of a continuum action imply certain relations for the corresponding lattice action. For general linear symmetries one can deduce a deformed symmetry relation for the lattice theory (4.1) that is a generalisation of the standard GW-relation.

However the construction of lattice symmetry operators for space-time dependent symmetries is subject to the obstruction (3.1). This constraint reflects the necessity to reconcile the averaging procedure of the blocking with the space-time structure of the symmetry. For space-time dependent symmetries it is non-trivial, which applies in particular to derivative-dependent symmetries such as supersymmetry, see (6.1), where we are led to the non-local SLAC-derivative in the lattice symmetry operator.

For well-defined symmetry operators on the lattice we have to require that both, the lattice operator $M_{\text {def }}$ and the corresponding lattice action are local. For non-local $M_{\text {def }}$ and/or lattice actions the fate of the symmetry in the continuum limit is unclear as lattice artefacts might survive.

Within this setting we have discussed supersymmetric theories at the example of supersymmetric quantum mechanics. We have shown that solutions can be achieved in this case with a quadratic action as well as in interacting theories. Although the symmetry relation (4.1) couples different orders of the fields, even for interacting theories polynomial solutions might be possible.

\section{References}

[1] P. H. Dondi and H. Nicolai, Nuovo Cim. A41 (1977) 1; M. Kato, M. Sakamoto and H. So, JHEP 05 (2008) 057.

[2] H. B. Nielsen and M. Ninomiya, Phys. Lett. B105 (1981) 219; D. Friedan, Commun. Math. Phys. 85 (1982) 481-490; O. Jahn and J. M. Pawlowski, Nucl. Phys. B 642 (2002) 357.

[3] P. H. Ginsparg and K. G. Wilson, Phys. Rev. D25, 2649 (1982).

[4] J. M. Pawlowski, Annals Phys. 322 (2007) 2831.

[5] G. Bergner, F. Bruckmann and J. M. Pawlowski, arXiv:0807.1110 [hep-lat].

[6] H. So and N. Ukita, Phys. Lett. B457 (1999) 314, [arXiv:hep-lat/9812002].

[7] M. Lüscher, Phys. Lett. B428 (1998) 342.

[8] S. D. Drell, M. Weinstein and S. Yankielowicz, Phys. Rev. D14 (1976) 487; S. D. Drell, M. Weinstein and S. Yankielowicz, Phys. Rev. D14 (1976) 1627.

[9] T. Kastner, G. Bergner, S. Uhlmann, A. Wipf and C. Wozar, arXiv:0807.1905 [hep-lat].

[10] Y. Igarashi, H. So and N. Ukita, Phys. Lett. B535 (2002) 363; Nucl. Phys. B640 (2002) 95. 